\title{
LA DISTRIBUCIÓN CONTINENTAL MÁS ORIENTAL DE Nothofagus antarctica EN EL RIOO GALLEGOS (SANTA CRUZ)
}

\author{
THE EASTERN CONTINENTAL DISTRIBUTION OF Nothofagus antarctica ALONG GALLEGOS \\ RIVER (SANTA CRUZ)
}

Pablo Luis Peri ${ }^{1,2}$, Guillermo Martínez Pastur ${ }^{3}$, Lucas Monelos ${ }^{2}$ \& Mario Beroiz ${ }^{4}$

El ñire o ñirre, Nothofagus antarctica (G. Forster) Oersted, es un árbol nativo que se caracteriza por su gran plasticidad fenotípica, adaptándose a una gran variedad de condiciones ambientales, ocupando desde sitios con exceso de humedad (turberas y mallines) hasta sitios muy secos (límite con la estepa) (Veblen et al., 1996; Rodríguez et al., 2005; Zuloaga, et al. 2008). Es considerada la especie de Nothofagus de Sudamérica con mayor variación morfológica (Ramírez et al., 1985) y de mayor tolerancia ecológica (Donoso et al., 2006). Esto determina una amplia distribución latitudinal $\left(36^{\circ} 50^{\prime}-56^{\circ} 00^{\prime}\right.$ S) desde el norte de Neuquén hasta el Cabo de Hornos. Altitudinalmente se distribuye desde el nivel del mar hasta los 2.000 m s.n.m. Sin embargo, en el sector continental argentino esta especie ocupa una estrecha franja cerca de la cordillera de los Andes, limitada por las precipitaciones (Veblen et al., 1996) y una baja capacidad de dispersión (por gravedad o viento) (Donoso et al., 2006) y corta viabilidad de sus semillas (Bahamonde et al., 2011) ocupando en la provincia de Santa Cruz una superficie de 159.720 ha (Peri \& Ormaechea, 2013). En este contexto, el objetivo del presente trabajo fue evaluar otras formas de dispersión del ñire a largas distancias. En primavera del 2012, se efectuó un relevamiento por los cursos de agua del Río Turbio y Río Gallegos través de la navegación con kayaks. El inicio del mismo fue desde la localidad de 28 de Noviembre (5136'21" S, 7211'54" W) ubicado en las cercanías de las masas boscosas continuas y puras de ñire, hasta la localidad de Río Gallegos (5137'20" S, 69¹3’01" W) a lo largo de aproximadamente $300 \mathrm{~km}$ de navegación. En cada relicto o grupo de individuos se tomaron fotografías, coordenadas con GPS, número de árboles, morfotipo, diámetro a la altura del pecho (DAP) máximos y alturas dominantes. En el presente trabajo un relicto de bosque nativo fue definido como aquel ecosistema forestal, primario o secundario maduro, compuesto principalmente por 


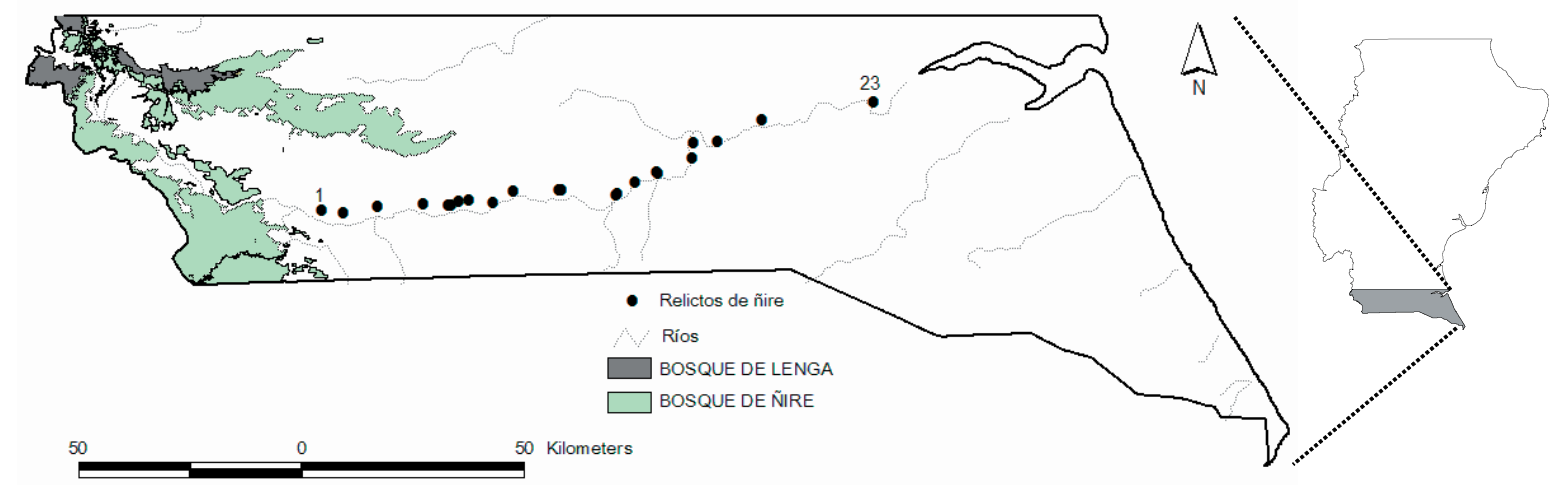

Fig. 1. Distribución de los relictos de ñire localizados a lo largo del Río Gallegos, provincia de Santa Cruz (Argentina). Desde el oeste hacia este por el cauce del río los círculos rojos representan en forma correlativa los relictos (R1 simboliza el primer relicto y R23 el último).

árboles nativos, que por diversos motivos (ya sea evolutivos, ontogénicos o biogeográficos) ha quedado lejos respecto de la masa continua de bosques de ñire conformando fragmentos de bosque diseminados en la orilla de los ríos, y poseen una superficie inferior a 1 ha. Se relevó un total de 23 relictos (Fig. 1) que se localizaron a orilla de los ríos entre los niveles inundables, en pequeñas islas o en las zonas de antiguos meandros. Se estima que la dispersión a larga distancia de esta especie está relacionada con eventos de crecida de los ríos, en el cual propágulos vegetativos (principalmente raíces) o árboles enteros fueron arrastrados por la corriente, determinando el éxito de su establecimiento y crecimiento la capacidad de anclaje y ubicación en el terreno del propágulo dispersado (Elosegi \& Díez, 2009). Si bien esta capacidad del ñire de propagarse en forma agámica ha sido registrada previamente a partir del rebrote de yemas de la porción basal del tallo luego de cortas o incendios, o rebrote de raíces, o brotes a partir de raíces adventicias de ramas parcialmente enterradas (Veblen et al., 1977), hasta el presente no existen registros para Patagonia argentina de eventos de propagación agámica a larga distancia por cauces de ríos.

Mientras que el primer relicto se ubica a $14 \mathrm{~km}$ desde la masa forestal continua más cercana de ñire, la distribución más oriental de ñire (5140'34,1" S, $69^{\circ} 43^{\prime} 06,4^{\prime \prime}$ W) se encuentra localizada a $32 \mathrm{~km}$ al oeste de la localidad de Río Gallegos, $53 \mathrm{~km}$ al oeste del océano Atlántico en línea recta y 180 km al este de la masa forestal continua más cercana de nire por el curso del río. La distancia promedio entre relictos por el cauce del río es de 7,4 km (máxima de 33,5 km y mínima de $50 \mathrm{~m}$ ). El tamaño de cada relicto fluctúa desde 1 a 180 árboles (Tabla 1). Ocho relictos están conformados por un individuo y el $82 \%$ posee menos de 10 árboles. El rango de DAP máximo promedio es de 2,5 a $39,7 \mathrm{~cm}$, y la altura máxima de árboles dominantes varía de 1,9 a 7,7 m (Tabla 1).

En la mayoría de los casos los árboles se establecieron en lugares abiertos, luminosos y con abundante agua en el perfil del suelo (Fig. 2), y donde los individuos poseen varios fustes (hasta 25 fustes por individuo) característicos de la propagación agámica por raíces (Stecconi, 2006). El morfotipo predominante es el arbóreo-arbustivo representado en 11 relictos (Tabla 1). Mientras que el morfotipo arbóreo tiene en general un eje principal definido, el arbóreo-arbustivo posee individuos con uno o varios troncos tortuosos con numerosas ramas laterales desarrolladas a partir de repetidos traumas ocasionados por los fuertes vientos. El morfotipo arbustivo achaparrado (2 relictos) forman matorrales compactos con un menor desarrollo en altura $(<$ $2,5 \mathrm{~m}$ ) y numerosos ejes comúnmente inclinados, lo cual evidencia una cierta inestabilidad del árbol por efectos combinados del viento, crecida del río y problemas de anclaje. El arbustivo (4 relictos) es un 
Tabla 1. Principales características de los relictos de ñire localizados a lo largo del Río Gallegos, provincia de Santa Cruz (Argentina).

\begin{tabular}{|c|c|c|c|c|c|c|c|}
\hline Relicto & Latitud & Longitud & $\begin{array}{c}\text { № } \\
\text { individuos }\end{array}$ & $\begin{array}{l}\text { DAP máximo } \\
(\mathrm{cm})\end{array}$ & $\underset{(\mathrm{m})}{\mathrm{h} \max }$ & $\begin{array}{l}\text { Morfotipo } \\
\text { predominante }\end{array}$ & $\begin{array}{l}\text { № fustes por } \\
\text { individuo }\end{array}$ \\
\hline $\mathrm{R} 1$ & $51^{\circ} 51^{\prime} 58,2^{\prime \prime}$ & $71^{\circ} 30^{\prime} 28,3^{\prime \prime}$ & 180 & 30,0 & 8,0 & arbóreo-arbustivo & 2 a 10 \\
\hline $\mathrm{R} 2$ & $51^{\circ} 52^{\prime} 23,4^{\prime \prime}$ & $71^{\circ} 26^{\prime} 21,2^{\prime \prime}$ & 1 & 4,7 & 1,9 & arbóreo-arbustivo & 14 \\
\hline R3 & $51^{\circ} 51^{\prime} 50,9^{\prime \prime}$ & $71^{\circ} 19^{\prime} 40,2^{\prime \prime}$ & 110 & 20,0 & 6,0 & arbustivo & 2 a 10 \\
\hline $\mathrm{R} 4$ & $51^{\circ} 51^{\prime} 42,1^{\prime \prime}$ & $71^{\circ} 10^{\prime} 42,9 ”$ & 1 & 2,9 & 2,0 & arbustivo achaparrado & $>30$ \\
\hline R5 & $51^{\circ} 51^{\prime} 55,4^{\prime \prime}$ & $71^{\circ} 05^{\prime} 48,7^{\prime \prime}$ & 6 & 4,0 & 2,4 & arbustivo achaparrado & 4 a 25 \\
\hline R6 & $51^{\circ} 51^{\prime} 55,1^{\prime \prime}$ & $71^{\circ} 05^{\prime} 45,9^{\prime \prime}$ & 4 & 18,4 & 4,3 & arbóreo-arbustivo & 10 a 25 \\
\hline R7 & $51^{\circ} 51^{\prime} 51,9^{\prime \prime}$ & $71^{\circ} 05^{\prime} 22,8^{\prime \prime}$ & 2 & 19,3 & 6,1 & arbóreo-arbustivo & 15 a 20 \\
\hline $\mathrm{R} 8$ & $51^{\circ} 51^{\prime} 30,4^{\prime \prime}$ & $71^{\circ} 03^{\prime} 45,5^{\prime \prime}$ & 18 & 5,2 & 2,5 & arbustivo & 4 a 10 \\
\hline R9 & $51^{\circ} 51^{\prime} 19,2^{\prime \prime}$ & $71^{\circ} 01^{\prime} 52,5^{\prime \prime}$ & 3 & 7,4 & 2,9 & arbustivo & 6 a 10 \\
\hline R10 & $51^{\circ} 51^{\prime} 44,2^{\prime \prime}$ & $70^{\circ} 57^{\prime} 14,3^{\prime \prime}$ & 2 & 24,4 & 5,0 & arbóreo & 1 a 4 \\
\hline R11 & $51^{\circ} 50^{\prime} 28,7^{\prime \prime}$ & $70 \div 53 ' 14,6 ”$ & 1 & 22,0 & 3,8 & arbóreo & 7 \\
\hline $\mathrm{R} 12$ & $51^{\circ} 50^{\prime} 22,8^{\prime \prime}$ & $70^{\circ} 44^{\prime} 10,8^{\prime \prime}$ & 1 & 39,7 & 7,5 & arbóreo & 8 \\
\hline $\mathrm{R} 13$ & $51^{\circ} 50^{\prime} 24,2^{\prime \prime}$ & $70^{\circ} 43^{\prime} 43,4^{\prime \prime}$ & 4 & 22,6 & 3,9 & arbóreo-arbustivo & 2 a 4 \\
\hline R14 & $51^{\circ} 51^{\prime} 10,2^{\prime \prime}$ & $70 \cong 33^{\prime} 05,1^{\prime \prime}$ & 1 & 9,3 & 4,0 & arbóreo-arbustivo & 7 \\
\hline R15 & $51^{\circ} 51^{\prime} 03,8^{\prime \prime}$ & $70^{\circ} 32^{\prime} 56,6^{\prime \prime}$ & 1 & 12,4 & 4,4 & arbóreo & 2 \\
\hline R16 & $51^{\circ} 49^{\prime} 45,7^{\prime \prime}$ & $70^{\circ} 29^{\prime} 34,1^{\prime \prime}$ & 2 & 16,4 & 6,4 & arbóreo & 4 \\
\hline R17 & $51^{\circ} 48^{\prime} 35,5^{\prime \prime}$ & $70^{\circ} 25^{\prime} 20,5^{\prime \prime}$ & 7 & 6,2 & 3,9 & arbóreo-arbustivo & 10 a 17 \\
\hline R18 & $51^{\circ} 48^{\prime} 36,8^{\prime \prime}$ & $70^{\circ} 25^{\prime} 12,1^{\prime \prime}$ & 2 & 6,7 & 4,4 & arbóreo-arbustivo & 12 a 15 \\
\hline R19 & $51^{\circ} 46^{\prime} 56,1^{\prime \prime}$ & $70^{\circ} 18^{\prime} 30,1^{\prime \prime}$ & 3 & 10,5 & 4,7 & arbóreo-arbustivo & 15 a 20 \\
\hline $\mathrm{R} 20$ & $51^{\circ} 45^{\prime} 00,9^{\prime \prime}$ & $70^{\circ} 18^{\prime} 12,9^{\prime \prime}$ & 1 & 12,1 & 6,2 & arbóreo-arbustivo & 16 \\
\hline $\mathrm{R} 21$ & $51^{\circ} 44^{\prime} 57,2^{\prime \prime}$ & $70^{\circ} 13^{\prime} 35,6^{\prime \prime}$ & 2 & 5,0 & 4,7 & arbóreo-arbustivo & 10 a 14 \\
\hline R22 & $51^{\circ} 42^{\prime} 29,5^{\prime \prime}$ & $70^{\circ} 04^{\prime} 48,8^{\prime \prime}$ & 25 & 15,0 & 7,7 & arbóreo & 4 a 20 \\
\hline R23 & $51^{\circ} 40^{\prime} 34,1^{\prime \prime}$ & $69^{\circ} 43^{\prime} 06,4^{\prime \prime}$ & 1 & 2,5 & 2,3 & arbustivo & 14 \\
\hline
\end{tabular}



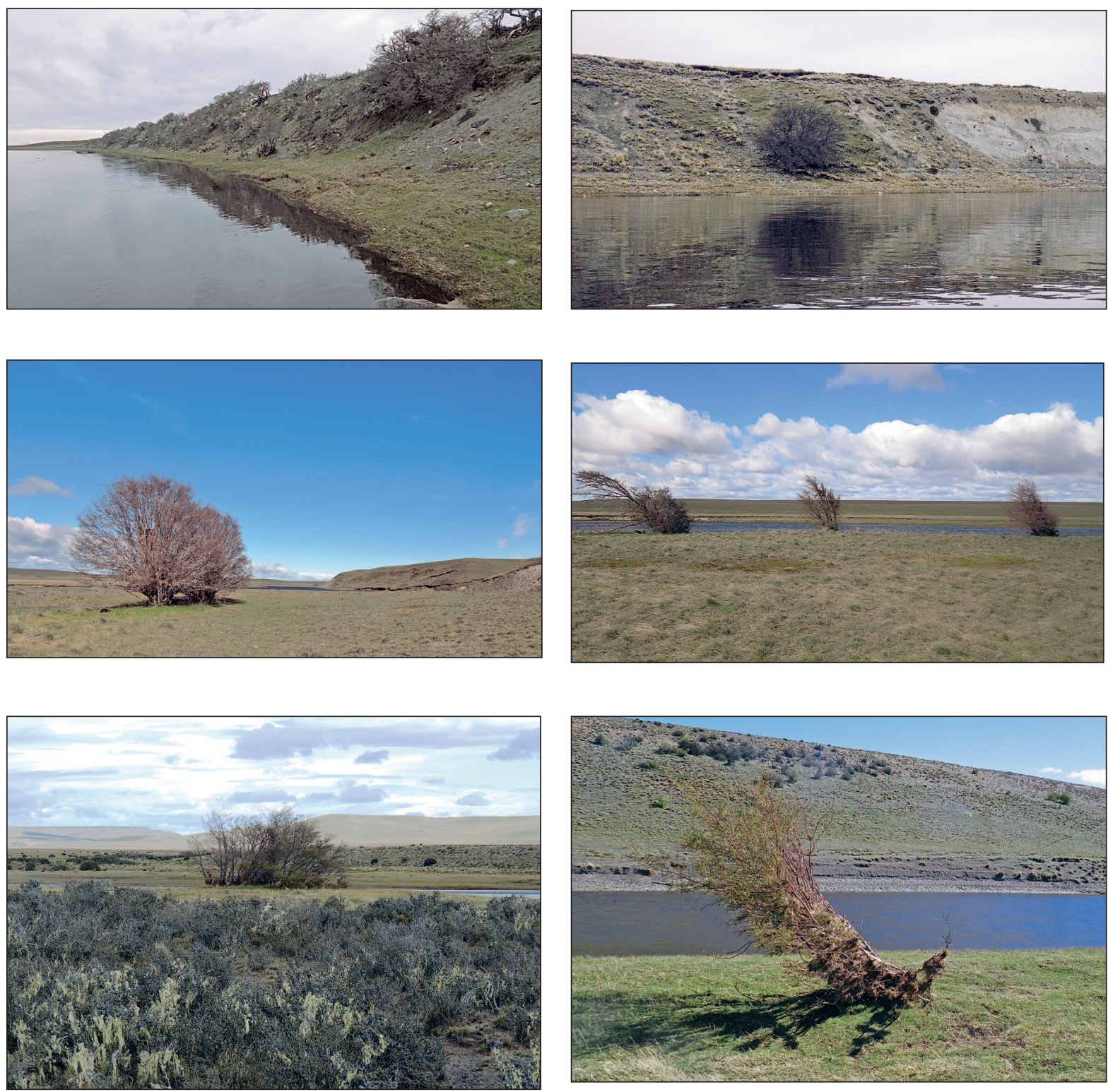

Fig. 2. A) Imagen del relicto $\mathrm{R} 1$ con el grupo de árboles más numeroso a orillas del Río Gallegos (Santa Cruz, Argentina)

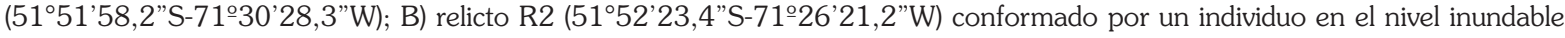

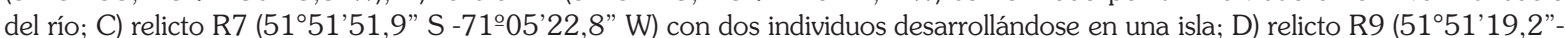

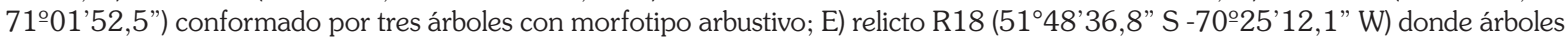
de ñire crecen rodeados del arbusto mata negra (Juniella tridens); F) último árbol de ñire (R23) creciendo en una isla y representando la distribución más oriental registrada para el ñire (5140'34,1" S -6943’06,4” W) localizado a 32 Km al oeste de la localidad de Río Gallegos en línea recta y $180 \mathrm{~km}$ al este de la masa forestal continua más cercana de ñire por el curso del río. 
morfotipo intermedio entre el arbóreo-arbustivo y arbustivo achaparrado en lo que respecta al desarrollo de altura y copa, y cuyos árboles tienen varios ejes verticales codominantes.

En varios sitios, los árboles de ñire comparten el mismo ambiente ribereño, y en algunos casos compiten, con individuos de sauce criollo (Salix humboldtiana Willd.) también propagados en forma agámica a lo largo del cauce del río. Además, es importante resaltar que la casi totalidad de los árboles maduros (>100 años) contenían nidos activos de caranchos (Caracara plancus Miller, 1777) y abundante presencia de egagrópilas. Esto demuestra el efecto facilitador de los árboles como hábitat para anidamiento de esta especie en un ambiente estepario.

Estos ambientes "relictuales" deberían ser considerados de muy alto valor de conservación donde los aspectos del grado de biodiversidad y salud de un bosque, toman mayor relevancia por tratarse de ecosistemas frágiles. La comprensión de la ecología de las especies nativas que conforman el ecosistema patagónico, además de proporcionar información útil para eventuales acciones de restauración, puede ser un aporte para hacer pronósticos relacionados con el la variación en el regimenes de cursos de agua debido al cambio climático. Para esta región los modelos climáticos predicen un aumento de temperatura (hasta $3^{\circ} \mathrm{C}$ ) y un incremento en la variabilidad de las precipitaciones para un período de 80 años (Kreps, et al. 2012). Sin embargo, es necesario profundizar el conocimiento de los relictos evaluados con estudios de genética, dendrocronología y biodiversidad asociada a estos ambientes.

\section{LITERATURA CITADA}

Bahamonde, H., Peri, P.L., Monelos L. \& G. Martínez Pastur 2011. Aspectos ecológicos de la regeneración por semillas en bosques nativos de Nothofagus antarctica en Patagonia Sur, Argentina. Bosque 32(1): 20-29.

Donoso, C., L. Steinke \& A. Premoli 2006. Nothofagus antarctica (G. Forster) Oerst. In: Donoso Zegers C. (Ed.) Las especies arbóreas de los bosques templados de Chile y Argentina. Autoecología. Marisa Cuneo Ediciones, Valdivia, Chile, 401-410.
Elosegi, A. \& J. Díez 2009. La vegetación terrestre asociada al río: el bosque de rivera. In: Elosegi A. \& S. Sabater (Eds.) Conceptos y técnicas en ecología fluvial. Fundación BBVA, España, 311-321.

Kreps, G., G. Martínez Pastur \& P.L. Peri 2012. Cambio Climático en Patagonia Sur: Escenarios futuros en el manejo de los recursos naturales Ediciones INTA, Buenos Aires: $100 \mathrm{pp}$.

Peri, P.L. \& S. Ormaechea 2013. Relevamiento de los bosques nativos de ñire (Nothofagus antarctica) en Santa Cruz: base para su conservación y manejo. Ediciones INTA, Buenos Aires.

Ramírez, C., M. Correa, H. Figueroa \& J. San Martín 1985. Variación del hábito y hábitat de Nothofagus antarctica en el sur de Chile. Bosque 6(2): 55-73.

Rodríguez, R., E. Ruíz \& J. Elissetche 2005. Árboles en Chile. Editorial Universidad de Concepción, Chile. 183pp.

Stecconi M. 2006. Variabilidad arquitectural de especies nativas de Nothofagus de la Patagonia (N. antarctica, N. pumilio, N. dombeyi). Tesis doctoral Universidad Nacional del Comahue (Centro Regional Universitario Bariloche), San Carlos de Bariloche, Río Negro, Argentina, 162 pp.

Veblen, T.T., D.H. Ashton, F.M. Schlegel \& A.T. Veblen 1977. Plant succession in a timberline depressed by vulcanism in south-central Chile. Journal of Biogeography 4: 275-294.

Veblen, T.T., C. Donoso, T. Kitzberger \& A.J. Rebertrus 1996. Ecology of Southern Chilean and Argentinean Nothofagus forests. In: Veblen T.T., R.S. Hill \& J. Read (Eds.) The ecology and biogeography of Nothofagus forests. Yale University Press, London, England, 293-353.

Zuloaga, F.O., O. Morrone, M.J. Belgrano (eds) 2008. Catálogo de las Plantas Vasculares del Cono Sur (Argentina, Sur de Brasil, Chile, Paraguay y Uruguay). Monographs of the Missouri Botanical Garden nº 107, vol. 2, Dicotyledoneae. 
